\title{
New Non-Quasi-Static Theory for Extracting Small-Signal Parameters Applied to LDMOSFETs
}

\author{
Patrick Roblin, Siraj Akhtar, and Jeffrey Strahler
}

\begin{abstract}
We present analytic formulas for simultaneously extracting the parasitic resistances, inductances, and the intrinsic parameters of a small-signal FET equivalent circuit model including the non-quasi-static (NQS) charging time-constants associated with the gate and drain charges, respectively. For the NQS equivalent circuit topology considered, there exists a continuum of solutions for the circuit parameters, as a function of the source resistance, giving exactly the same frequency response fit. A multi-bias analysis is used to determine the final source resistance. Realistic results are obtained for power LDMOSFETs despite the very small value of the parasitics in these power RF devices.
\end{abstract}

Index Terms-FET equivalent circuits, LDMOSFET, microwave small-signal modeling, non-quasi-static, parameter extraction.

\section{INTRODUCTION}

$\mathbf{P}$ ACKAGED Silicon power LDMOSFET transistors are finding important applications in RF power amplifier design, creating a demand for accurate models for use in circuit simulation [1], [2]. Efficient techniques have been developed for the extraction of the parasitics in quasi-static (QS) models [3], [4]. However, the extraction of $R_{g s i}$ and drain time-delay $\tau$ in non-quasi-static (NQS) models are known to be "notoriously unstable," and "show enormous sensitivities" as stated by King et al. [5]. An iterative method has been recently proposed by Raskin et al. [6]. In this letter, we present analytic formulas for a charge-based model topology relying on two NQS time-constants [7], [8].

\section{NEW EXTRACTION THEORY}

At microwave frequencies, the iso-thermal microwave response of the LDMOSFET can be well represented by the NQS small-signal model shown in Fig. 1, where the intrinsic $Y$-parameters are: $Y_{i n t r, i j}=g_{i j}+j \omega C_{i j} /\left(1+j \omega \tau_{i j}\right)$ with $g_{11}=g_{12}=0, g_{22}=g_{d}$ and $g_{21}=g_{m}$. In agreement with its associated charge-based NQS large-signal model topology [7] we limit our analysis to a model with two NQS time-constants: $\tau_{G}=\tau_{12}=\tau_{11}$ for the gate charge and $\tau_{D}=\tau_{22}=\tau_{21}$ for the drain charge.

In the presence of resistive and inductive parasitics at the terminals, one verifies that the extrinsic $Z_{i j}$ for the NQS model

Manuscript received April 10, 2000; revised June 5, 2000.

P. Roblin and S. Akhtar are with the Department of Electrical Engineering, The Ohio State University Columbus, OH 43210 USA.

J. Strahler is with the Lucent Technologies Columbus, OH 43213 USA.

Publisher Item Identifier S 1051-8207(00)07039-2. selected assumes a frequency dependence similar to that of the QS model [3], [4]

$$
\begin{aligned}
\operatorname{Re}\left[Z_{e x t r, i j}\right] & =R_{i j}+\frac{A_{i j}}{\omega^{2}+B_{r}^{2}} \\
\frac{1}{\omega} \operatorname{Im}\left[Z_{e x t r, i j}\right] & =L_{i j}+\frac{B_{i j}}{\omega^{2}+B_{r}^{2}}+\frac{G_{i j}}{\omega^{2}\left(\omega^{2}+B_{r}^{2}\right)}
\end{aligned}
$$

where the various coefficients $R_{i j}$ are now given by

$$
\begin{aligned}
& R_{12}=R_{s}+\delta R_{12} \quad \text { with } \delta R_{12}=\frac{C_{12} \tau_{D}}{A} \\
& R_{22}=R_{s}+R_{d}+\delta R_{22} \quad \text { with } \delta R_{22}=-\frac{C_{11} \tau_{D}}{A} \\
& R_{11}=R_{s}+R_{g}+\delta R_{11} \quad \text { with } \delta R_{11}=-\frac{\tau_{G} \tau_{D} g_{d}+C_{22} \tau_{G}}{A} \\
& R_{21}=R_{s}+\delta R_{21} \quad \text { with } \delta R_{21}=\frac{\tau_{G} \tau_{D} g_{m}+C_{21} \tau_{G}}{A}
\end{aligned}
$$

with $A=C_{12} C_{21}-C_{11} C_{22}+\tau_{D}\left(g_{m} C_{12}-g_{d} C_{11}\right)$ and $\delta R_{i j}$ the NQS corrections.

One can then derive the formulas given in Table I for calculating the extrinsic and intrinsic model parameters in terms of the known fit-parameters $A_{i j}, R_{i j}, L_{i j}, B_{r}^{2}$, and $G_{i j}$ [3] and the unknown source resistance $R_{s}$. For this two- $\tau$ NQS model, there exists therefore a continuum of solutions as a function of $R_{s}$ which yield the exact same fit to the $Z_{i j}$ or $S_{i j}$ parameters. However, note that the capacitances $C_{11}$ and $C_{12}$, the extrinsic $g_{D}$ and $g_{M}$, and the parasitic inductances $L_{s}, L_{g}$, and $L_{d}$ are independent of $R_{s}$ and hence uniquely extracted.

\section{EXPERIMENTAL RESULTS}

For this work we have used Motorola's MRF 181 RF power, $n$-channel enhancement mode, lateral MOSFET [9]. Isothermal microwave data were acquired for various bias points and the text fixture contribution deembedded using a TRL calibration procedure.

First the regression procedure of Raskin et al. [4] was used to establish the linear relationships giving the $R_{i j}$ and $L_{i j}$ coefficients in terms of $R_{11}$ and $L_{12}$, respectively. Next the independent coefficients $R_{11}, A_{i j}$ and $B_{r}^{2}$ were obtained from a simultaneous least-square-fit of all the $\operatorname{Re}\left[Z_{i j}\right]$. Finally, $G_{21}$ and $L_{12}$ were obtained from a simultaneous fit of all the $\operatorname{Im}\left[Z_{i j}\right]$ using in addition the theoretical model constraints: $A_{i j}= \pm g_{i j} / A+$ $B_{r} B_{i j}$. A typical fit obtained is shown in Fig. 2.

Once the extrinsic parameters $Z_{e x t r, i j}$ are fitted, the extrinsic and intrinsic model parameters are calculated as a function of $R_{s}$ using the expressions given in Table I. Fig. 3 shows 


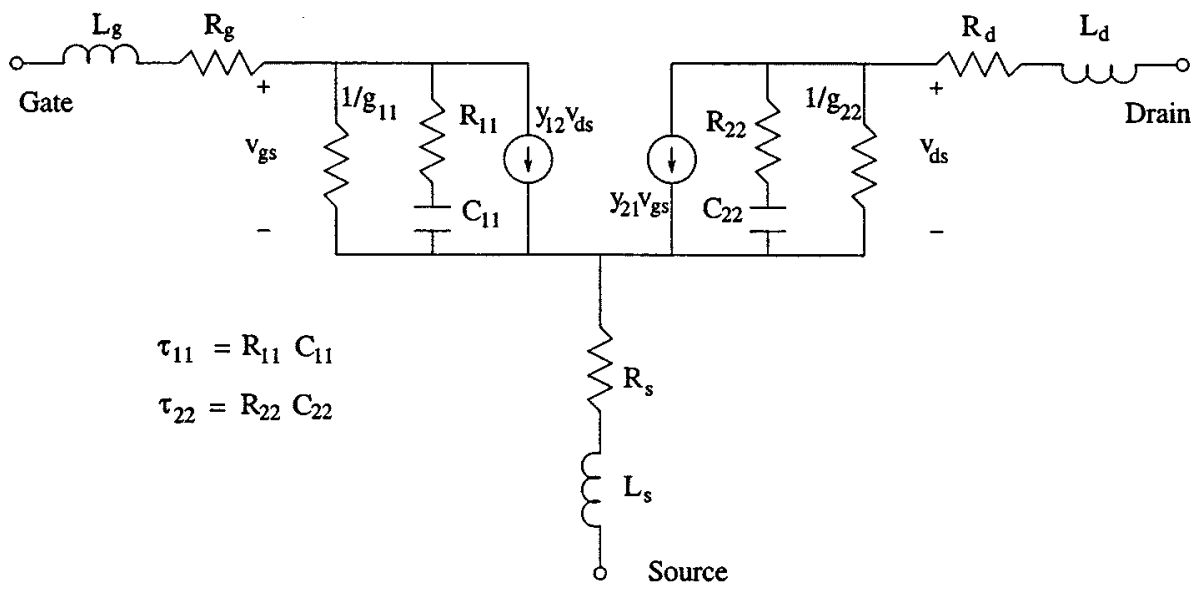

Fig. 1. NQS small-signal equivalent-circuit for the high-frequency response

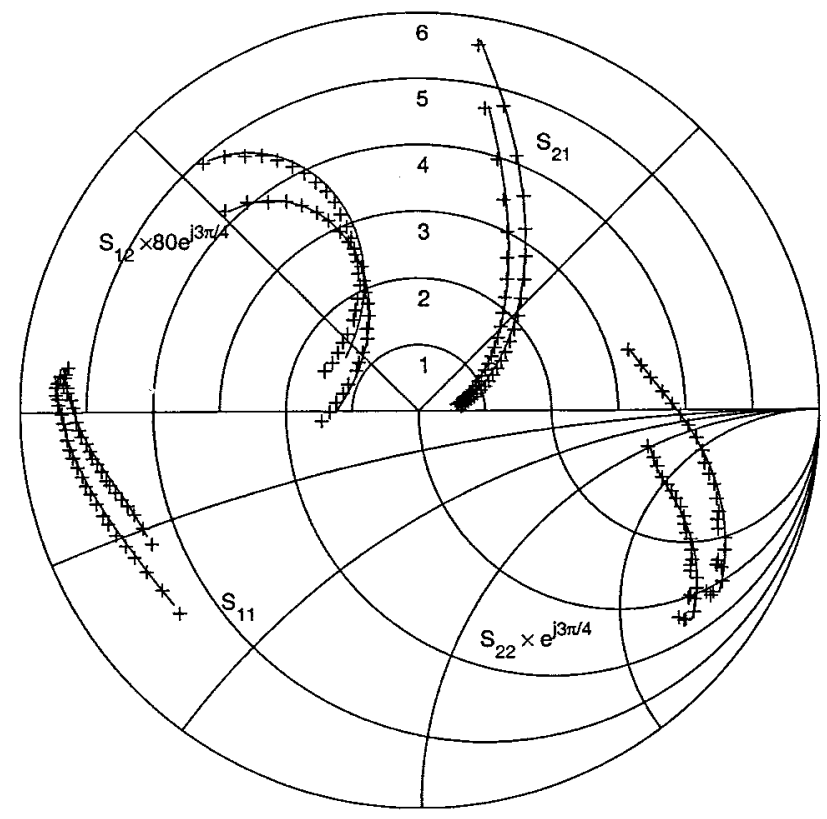

Fig. 2. Extrinsic $S$-parameters measured from $0.4-2 \mathrm{GHz}$ for 2 different bias points in saturation $\left(V_{G S}=4\right.$ and $\left.4.75 \mathrm{~V}\right)$.

for different biases ( $V_{G S}, V_{D S}$ ), the extracted $R_{d}$ and $R_{g}$ as functions of $R_{s}$. The $R_{d}$ and $R_{g}$ loci are limited to values of $R_{s}$ giving the correct physical sign for all parameters. Note that the bias points were selected to be in the saturation region. The same line-symbol is used for curves with the same $V_{G S}$. The loci of $R_{d}$ versus $R_{s}$ indicate that a common $R_{d}$ value is obtained for $R_{s}=0.136 \pm 0.002 \Omega$ for all gate and drain voltages. The drain resistance at the intercept point slightly increases from $R_{d}\left(V_{G S}=4 V, V_{D S}\right)=0.67 \pm 0.005 \Omega$ to $R_{d}\left(V_{G S}=4.75 V, V_{D S}\right)=0.785 \pm 0.015 \Omega$ as the gate voltage varies from $4-4.75 \mathrm{~V}$ and is fairly insensitive to the drain voltage. This indicates that in saturation, the drain resistance is effectively a nonlinear function of the saturation drain-current. No intercept is found from the $R_{g}$ loci.
TABLE I

Model Parameters In Terms of the $Z_{i j}$ Fit Parameters

\begin{tabular}{|c|c|}
\hline$L_{s}=L_{12}=L_{21}$ & $L_{g}=L_{11}-L_{s}$ \\
\hline$L_{d}=L_{22}-L_{s}$ & $B_{r}=\frac{B_{11} B_{r}^{2}-G_{11}}{A_{11}}$ \\
\hline$C_{11}=\left\{-\frac{G_{11}}{B_{r}^{2}}+\frac{A_{12}}{A_{22}} \frac{G_{21}}{B_{r}^{2}}\right\}^{-1}$ & $C_{12}=\left\{-\frac{Q_{21}}{B_{r}^{2}}+\frac{A_{22}}{A_{12}} \frac{G_{11}}{B_{r}^{2}}\right\}^{-1}$ \\
\hline$g_{M}=\frac{-G_{21}}{G_{11}\left(R_{22}+A_{22} / B_{r}^{2}\right)-\left(f_{21}\left(R_{12}+A_{12} / B_{r}^{2}\right)\right.}$ & $g_{D}=\frac{G_{11}}{\left(i_{11}\left(R_{22}+A_{22} / B_{r}^{2}\right)-\left(G_{21}\left(R_{12}+A_{12} / B_{r}^{2}\right)\right.\right.}$ \\
\hline$R_{d}=\left(-1+\frac{A_{22}}{A_{12}}\right) R_{s}+\left(R_{22}-R_{12} \frac{A_{22}}{A_{12}}\right)$ & $\tau_{D}=\frac{R_{s}-R_{12}}{B_{r}\left(R_{12}-R_{s}+A_{12} / B_{r}^{2}\right)}$ \\
\hline$c_{11 p}=-\frac{A_{22}}{A_{12}} R_{s}+\left[\frac{A_{22}}{B_{*}^{2}}+R_{12} \frac{A_{22}}{A_{12}}\right]$ & $c_{12 p}=R_{s}-R_{12}-\frac{A_{12}}{B_{v}^{2}}$ \\
\hline$B=-\frac{B_{x}^{2}}{c_{11 p} G_{11}+c_{12 p} G_{21}}$ & $R_{p 21}=\left(R_{s}-R_{21}\right) \frac{B}{B_{r}}$ \\
\hline$g_{m}=\frac{B G_{21}}{B_{f}^{2}}$ & $g_{d}=-\frac{B G_{11}}{B_{r}^{2}}$ \\
\hline$I_{p 21}=B_{21} \frac{B}{B_{r}}+B\left(R_{21}-R_{s}\right)$ & $\tau_{G}=-\frac{I_{\rho 21}}{2 g_{m}} \pm\left(\left(\frac{I_{p 21}}{2 g_{m}}\right)^{2}+\frac{R_{p 21}}{g_{m}}\right)^{1 / 2}$ \\
\hline$C_{21}=-\left[I_{p 21}+\left(\tau_{D}+\tau_{G}\right) g_{m}\right]$ & $C_{22}=-\frac{B}{C_{11}}\left(\frac{1}{B_{r}}-\frac{C_{12} C_{21}}{B}+\tau_{D}\right)$ \\
\hline$R_{p 11}=\tau_{G} \tau_{D} g_{d}+\tau_{G} C_{22}$ & $R_{g}=R_{11}-R_{s}+\frac{R_{p 11} B_{r}}{B}$ \\
\hline
\end{tabular}

The absence of intercept originates from both the sensitivity of the extracted $R_{g}$ loci to the data noise in the measured $S$-parameters and the weak dependence of $R_{g}$ upon $R_{s}\left(R_{g}\right.$ loci are horizontal). From the vertical distribution of $R_{g}$ loci we estimate that the average value of the gate resistance is $R_{g}=0.75 \pm 0.05 \Omega$ which still provides an acceptable margin of error.

The values for the parasitics using the QS regression method [4] were verified to correlate well with the NQS analysis, however the present analysis also yields the NQS time-constants: $\tau_{G}=1.830 \mathrm{ps}$ and $\tau_{D}=0.668 \mathrm{ps}$ which are known to be difficult to extract [5]. Note that $\delta R_{21}$ is in average $42 \%$ of $R_{21}$. On the other hand, $\delta R_{12}$ is in average only $5.6 \%$ of $R_{12}$ so that for the particular device considered here, the assumption $R_{s}=R_{12}$ instead of the multi-bias analysis, could have alternately been used in the equations of Table I for calculating all the remaining NQS model parameters. 


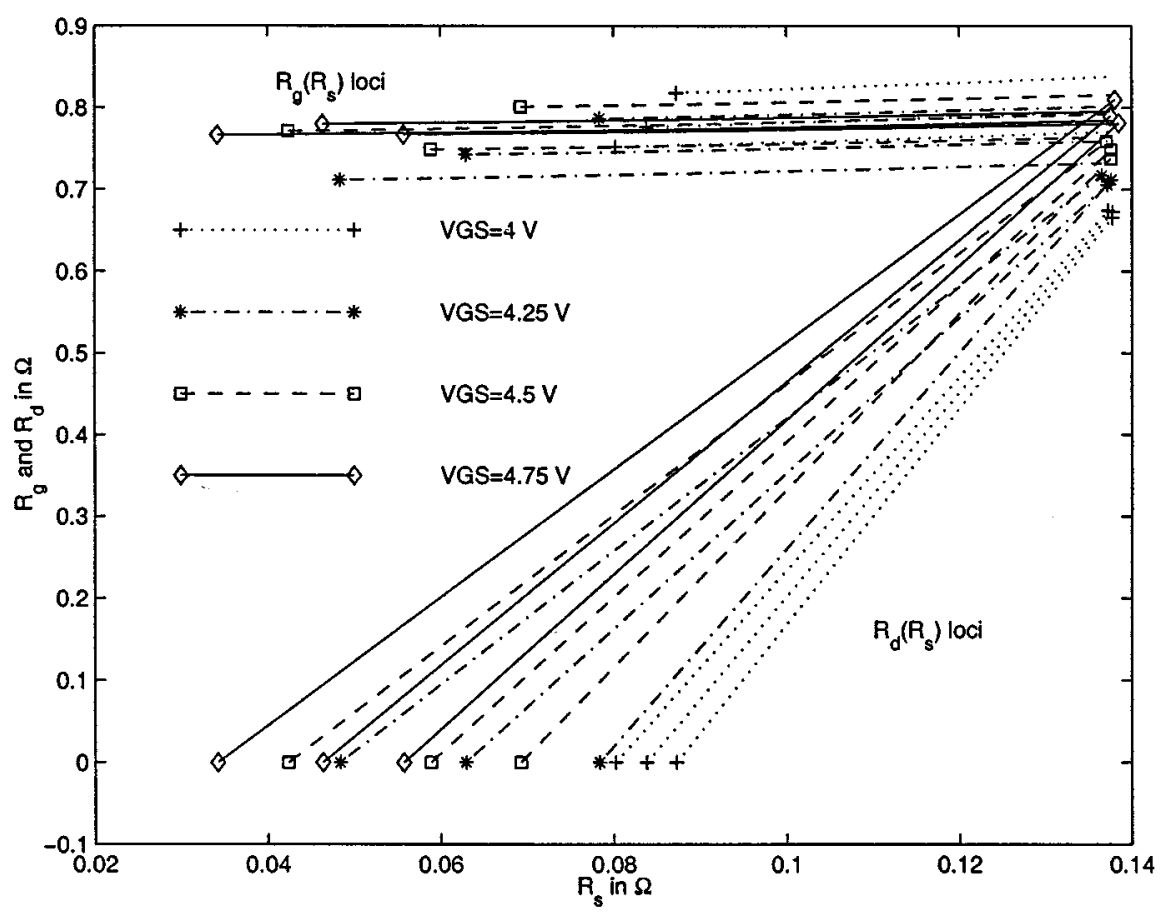

Fig. 3. Loci of $R_{d}$ and $R_{g}$ as a function of $R_{s}$ for various gate and drain voltages. The same line-symbol is used for the loci of different $V_{D S}$ with the same $V_{G S}$. Note that the loci are limited to values of $R_{s}$ giving the correct physical sign for all parameters. The source resistance at all the $R_{d}$ intercept point is about $R_{s}=0.136 \Omega$. The drain resistance $R_{d}$ at the $R_{d}$ intercept point slightly increases from 0.67 to $0.785 \Omega$ as $V_{G S}$ increases from 4 to $4.75 \mathrm{~V}$.

\section{CONCLUSION}

We have presented a new extraction theory which provides analytic formulas to extract the two time-constants of a NQS model simultaneously with all the parasitics and other intrinsic model parameters. It is to be noted, however, that the assumption $\tau_{12}=\tau_{11}$ and $\tau_{21}=\tau_{22}$ used by the charge-based NQS model selected might not be necessarily applicable to all FETs. However, realistic results were obtained for various LDMOSFETs in spite of the very small values of the parasitics resistances in these power devices.

\section{ACKNOWLEDGMENT}

The authors thank Prof. Raskin for many interesting and useful discussions and the reviewers for useful suggestions.

\section{REFERENCES}

[1] Collantes, J. J. Raoux, R. Quere, and A. Suarez, "New measurementbased technique for RF LDMOS nonlinear modeling," IEEE Microwave Guided Wave Lett., vol. 8, pp. PAGE NOS?-, Oct. 1998.
[2] W. R. Curtice, J. A. Pla, Bridges, T. Liang, and E. E. Shumate, "A new dynamic electro-thermal nonlinear model for silicon RF LDMOS FETs," Microwave Symp. 99, vol. 2, pp. 419-422, 1999.

[3] S. L. Lee, H. K. Yu, C. S. Kim, J. G. Koo, and K. S. Nam, "A novel approach to extracting small-signal model parameters of Silicon MOSFET's," IEEE Microwave Guided Wave Lett., vol. 7, Mar. 1997.

[4] J. P. Raskin, G. Dambrine, and R. Gillon, "Direct extraction of the series equivalent circuit parameters for the small-signal model of SOI MOSFET's," IEEE Microwave Guided Wave Lett., vol. 7, pp. 408-410, Dec. 1997.

[5] F. D. King, P. Winson, A. D. Snider, L. Dunleavy, and D. P. Levinson, "Math methods in transistor modeling: Condition numbers for parameter extraction," IEEE Trans. Microwave Theory Tech., vol. 46, pp. $1313-1314$, Sept. 98

[6] J.-P. Raskin, R. Gillon, J. Chen, D. Vanhoenacker-Janvier, and J.-P. Colinge, "Accurate SOI MOSFET characterization at microwave frequencies for device performance optimization and analog modeling," IEEE Trans. Microwave Theory Tech., vol. 45, pp. 1017-1025, May 1998.

[7] P. Roblin, S. C. Kang, and W. R. Liou, "Improved small-signal equivalent circuit model and large-signal state-equations for the MOSFET/MODFET wave equation," IEEE Trans. Electron Devices, vol. 38, pp. 1706-1718, Aug. 1991.

[8] S. Akhtar and P. Roblin, "B-spline based, large-signal DC and microwave-model for SOI-MOSFETs," Analog Integr. Circuits Signal Process., to be published.

[9] Motorola, "The RF MOSFET Line: RF Power Field Effect Transistor: $N$-Channel Enhancement-Mode Lateral MOSFET," Motorola Semiconductor Technical Data. 